\title{
Pathogenetic Mechanism of Type 2 Diabetes Mellitus and its Clinical Implications
}

\author{
Mala Dharmalingam Sara Rani Marcus² \\ ${ }^{1}$ Department of Endocrinology, M.S. Ramaiah Medical College, \\ Bengaluru, India \\ 2Department of Biochemistry, M.S. Ramaiah Medical College, \\ Bengaluru, India
}

\begin{abstract}
Address for correspondence Mala Dharmalingam, MD, DM, Department of Endocrinology, M.S. Ramaiah Medical College, New BEL Road, Mathikere, Bengaluru 560054, India (e-mail: drmaladharmalingam@gmail.com).
\end{abstract}

Ann Natl Acad Med Sci (India) 2019;55:132-134

\begin{abstract}
Keywords

- oxidative stress

- diabetes mellitus

- obesity

- anemia

Oxidative stress is an important pathogenetic mechanism for the development of type 2 diabetes mellitus (T2DM) and its complications. Oxidative stress is an imbalance of the generation of free radicals (reactive oxygen species [ROS] and reactive nitrogen species [RNS]) and their neutralization by the antioxidant mechanisms. Increased levels of ROS and RNS lead to damage of lipids, proteins, and DNA, ultimately causing the destruction of the islet cells of pancreas through apoptosis. Another important factor in the development of diabetes mellitus and metabolic syndrome is inflammation. We studied oxidative stress in type 2 diabetic patients, patients with obesity, metabolic syndrome, and T2DM with iron-deficiency anemia. The elevation of oxidative stress in these conditions along with the increase in inflammation suggests that both oxidative stress and inflammation may heighten the risk for the development of T2DM and its complications.
\end{abstract}

\section{Introduction}

Type 2 diabetes mellitus (T2DM) is a rapidly increasing problem in India. The prevalence has been estimated at $8.8 \%{ }^{1}$ This has been attributed to an underlying high genetic risk, which is abetted by the environmental factors of obesity, lifestyle, and stress. The role of the initiating factors like oxidative stress and inflammation has been studied in T2DM. The risk factors of the metabolic syndrome, which is one of the main pathogenetic factors apart from insulin deficiency, should be the target to address before the onset of glucotoxicity and lipotoxicity. Metabolic syndrome encompasses a cluster of risk factors like glucose intolerance, hyperinsulinemia, dyslipidemia, and clinical features such as increased abdominal circumference, acanthosis nigricans, and polycystic ovarian disorder. Variations in the definitions have led to difficulties in determining the prevalence of metabolic syndrome in the population. $^{2}$

In the progression of metabolic syndrome to T2DM, there are inherent genetic factors, like polymorphism of the apolipoprotein E4, aldose reductase, angiotensin converting enzyme, and toll receptor genes. The initiating events include inflammation, oxidative stress, activation of protein kinase C, selectins, vascular cell adhesion molecules and interleukins, tumor necrosis factor $\alpha$ and nuclear factor kappa $\beta$ (NF-KB) and nitrotyrosine. If these initiating events are not targeted early, they progress to functional and pathologic changes of lipotoxicity and glucotoxicity to diabetes and its complications. ${ }^{3}$

Oxidative stress is a state in which there is an excessive generation or incomplete removal of reactive oxygen radicals (ROS) and reactive nitrogen species (RNS), resulting in the damage to cellular macromolecules by these reactants. The ROS include superoxide, hydrogen peroxide, hydroxyl radicals, and singlet oxygen and RNS include nitric oxide and peroxynitrite. In a healthy human being, the endogenous sources of oxidative stress include mitochondria, peroxisomes, lipoxygenase, nicotinamide adenine dinucleotide phosphate oxidase, and cytochrome P450 system; the exogenous sources include ultraviolet light, ionizing radiation, chemotherapeutics, inflammatory cytokines, and environmental toxins. ${ }^{4}$ Low levels of ROS act as physiological and normal cell signaling molecules that mediate cellular differentiation, survival, and metabolism. ${ }^{5}$ However, increased levels of oxidative stress lead to impaired physiological conditions of 
decreased proliferative response and defective host response. A balance is maintained by the antioxidant defenses of enzymatic systems of catalase (CT), glutathione peroxidase, superoxide dismutase, and nonenzymatic systems of glutathione, vitamins $\mathrm{A}, \mathrm{C}$, and $\mathrm{E}$ and molecules like uric acid and bilirubin. ${ }^{5}$ If the antioxidant mechanism is defective then there is cellular damage and specific signaling pathways are affected leading to aging, disease, and cell death.

\section{T2DM, Oxidative Stress, and Inflammation}

The fundamental abnormality in T2DM is hyperglycemia, which is associated with oxidative stress. The metabolic pathways induced by hyperglycemia are: the polyol pathway, the hexosamine pathway, activation of protein kinase $C$, and the advanced glycation end products pathway; alterations in these pathways cause enhanced oxidative stress. ${ }^{6}$ Increased ROS lead to the damage of DNA, lipids, and proteins. As the diabetes progresses, the ROS can cause $\beta$-cell failure and insulin resistance. $\beta$-cells are particularly susceptible to ROS due to low expression of antioxidants like catalase and superoxide dismutase $2 .^{7}$ Oxidative stress at the cellular level leads to pancreatic islet cell damage as well as the microvascular complication of the eyes, nerves, and kidney.

Oxidative stress and inflammation associated with obesity and metabolic syndrome probably contribute to the progression of the clinical manifestations of the metabolic syndrome. ${ }^{8}$ Insulin has been shown to exhibit anti-inflammatory activity: it suppresses several proinflammatory transcription factors like NF-кB, Egr-1, and activating protein-1 and their corresponding genes that mediate inflammation. ${ }^{8}$ However, insulin resistance would cause the activation of these transcription factors leading to inflammation.

\section{Oxidative Stress in T2DM}

To understand oxidative stress in diabetes and the link between hyperglycemia and enhanced free radical activity, we studied the lipid peroxidation and protein carbonyl levels in diabetics. We studied 60 diabetics and age- and sexmatched controls. Malondialdehyde, measured as thiobarbituric acid reactive substances; an index of lipid peroxidation) and protein carbonyl levels (index of protein damage by free radicals), was studied and compared with normal healthy individuals. Both malondialdehide (MDA) and protein carbonyl levels were significantly increased in the type 2 diabetics. The enhanced lipid peroxidation leads to increase in free radical activity and together with protein damage increases insulin resistance and cell damage. ${ }^{9,10}$

\section{Obesity, Metabolic Syndrome, and Oxidative Stress}

Obesity, a growing problem in India and the world over, is associated with oxidative stress and low-grade inflammation. ${ }^{11}$ We studied oxidative stress in obesity.

Obese patients were divided into two groups of obesity and metabolic syndrome and compared with 30 age- and sex-matched controls. As expected, weight, waist and hip circumference, and the waist hip ratio were more in patients with obesity and metabolic syndrome. The systolic and diastolic blood pressure was also high in patients with metabolic syndrome. In the metabolic parameters, the triglyceride and very low density lipoproteins were significantly more in both the obese and the metabolic syndrome groups and there was a trend of increasing cholesterol in the metabolic syndrome group but was not significant. We measured lipid soluble and water soluble hydroperoxides and the total antioxidant capacity. While the water soluble hydroperoxide level was significantly increased in the obese and metabolic syndrome patients, the lipid soluble hydroperoxides and total antioxidant capacity did not show any change. ${ }^{12}$ In another study, the levels of high-sensitivity C-reactive protein (hs-CRP), a nonspecific marker of inflammation was measured in obese women and compared with nonobese age-matched controls. The enhanced levels of hs-CRP in obese women indicate an increase in the levels of inflammation. ${ }^{11}$ We concluded that early identification of at-risk obese patients by markers like increased oxidative stress, insulin resistance, inflammation, dyslipidemia, and some anthropometric parameters may decrease the progression of complications of metabolic syndrome.

\section{T2DM, Anemia, and Oxidative Stress}

Iron-deficient anemia is a common global nutritional disorder highly prevalent in the developing countries. There is a close association between the metabolisms of iron and glucose: while insulin is required for the uptake of iron in cells, in the liver, iron influences glucose metabolism and the uptake and metabolism of insulin. Iron plays a direct and causal role in diabetes pathogenesis by mediating both $\beta$-cell failure and insulin resistance. Iron $\left(\mathrm{Fe}^{2+}\right)$ is a prooxidant and catalyzes the generation of highly reactive hydroxyl radical, resulting in damage to cellular macromolecules. Iron overload states increase the incidence of T2DM, which may be reversed by the reduction of iron load. ${ }^{13}$ Oxidative stress due to the prooxidant role of iron may contribute to tissue damage and enhance the risk for diabetes. ${ }^{13}$ Iron-containing proteins like catalase and peroxidase function as antioxidants and reduction in the levels of iron would impair the antioxidant defense system. Hence, the role of iron on oxidative stress in iron deficiency together with diabetes was studied. We studied two groups of 30 patients, each having diabetes with and without iron deficiency, and compared them to normal individuals. We studied the parameters of anemia, namely, iron profile, and markers of oxidative stress (MDA levels as a marker of lipid peroxidation and serum uric acid levels)..$^{14}$ Serum iron was significantly low in the patients with iron deficiency anemia. A significant increase in the malondialdehyde levels and decrease in the uric acid levels in the iron-deficient diabetic patients when compared with diabetics without iron deficiency, were observed. We concluded that diabetes is a state of lower antioxidant defenses because in iron deficiency the enzymes involved in the antioxidant defense system would be functionally defective due 
to decreased levels of iron-containing enzymes like catalase and peroxidase, which are important free radical scavengers. ${ }^{15}$ Thus, iron deficiency also results in enhanced oxidative stress. Elevated serum ferritin levels observed in diabetes without iron deficiency suggest the increase in inflammation. Increased oxidative stress and inflammation progress to the development of T2DM and its complications.

\section{Discussion}

There are many studies on diabetes and oxidative stress in the world literature. ${ }^{16}$ The association of obesity and oxidative stress has also been studied. Their findings are similar to our studies and they have found oxidative stress to be increased in obese (independent of glycemic intolerance) and diabetic patients. Although oxidative stress with iron overload has been studied extensively, there are few reports of oxidative stress in iron-deficiency anemia. ${ }^{15} \mathrm{We}$ have found that iron-deficiency with diabetes also leads to enhanced oxidative stress due to decreased antioxidant levels.

In the presence of hyperglycemia, there is enhanced oxidative stress in several tissues. The ROS, in turn, activate stress sensitive signaling pathways that regulate gene expression resulting in the deterioration of the islets $\beta$-cells of the pancreas, thus resulting in reduced release of insulin. ${ }^{17}$ Also, interference with the insulin signaling pathways results in the development of insulin resistance. ${ }^{18}$

Another risk factor of diabetes mellitus is inflammation. ${ }^{17}$ The inflammatory condition triggers the development of insulin resistance and diabetes mellitus through a very complex mechanism consisting of several kinases and signaling pathways. ${ }^{18}$ Mechanistically, the adipocytes and immunocytes produce various proinflammatory cytokines including interleukin- 6 (IL-6) and tumor necrosis factor- $\alpha$ (TNF- $\alpha$ ) that are involved in the pathogenesis of diabetes mellitus. ${ }^{19}$ These cytokines are involved in the activation of the NF- $\mathrm{kB}$ pathway leading to serine phosphorylation of insulin receptor substrate resulting in the insulin resistance. ${ }^{20}$ Further, diabetes is also induced by the islets $\beta$-cells dysfunctioning caused by excessive IL-6 and TNF- $\alpha$. $^{21}$

\section{Conclusion}

Oxidative stress and inflammation are important pathogenetic factors in the initiation of diabetes and its complications. To control the epidemic of diabetes in our country we will have to look at the factors generating oxidative stress for primary prevention of diabetes and secondary prevention of complication of diabetes.

\section{Note}

Mala Dharmalingam was selected for Dr. J.S. Bajaj Oration for the year 2018-2019.

\section{Conflict of Interest}

None declared.

\section{References}

1 Diabetes Atlas, 8th ed., Global Fact Sheet. Brussels, Belgium: International Diabetes Federation; 2017

2 Kempegowda P, Marcus SR, Solanki P, Reddy RS, Nandini DR, Dharmalingam M. Prevalence of the metabolic syndrome in rural India-a disparity in definitions. Int J Diabetes Dev Countries 2011;31(4):188-193

3 Vinik AI, Mehrabyan A. Diabetic neuropathies. Med Clin North Am 2004;88(4):947-999

4 Vasudevan DM, Sreekumari S, Textbook of Biochemistry. 3rd ed. New Delhi: Jaypee Brothers Medical Publishers (P) Ltd; 2001:338-339

5 Roberts CK, Sindhu KK. Oxidative stress and metabolic syndrome. Life Sci 2009;84(21-22):705-712

6 Brownlee M. The pathobiology of diabetic complications: a unifying mechanism. Diabetes 2005;54(6):1615-1625

7 Azevedo-Martins AK, Lortz S, Lenzen S, Curi R, Eizirik DL, Tiedge M. Improvement of the mitochondrial antioxidant defense status prevents cytokine-induced nuclear factor-kappa B activation in insulin-producing cells. Diabetes 2003;52(1):93-101

8 Dandona P, Aljada A, Chaudhuri A, Mohanty P, Garg R. Metabolic syndrome: a comprehensive perspective based on interactions between obesity, diabetes, and inflammation. Circulation 2005;111(11):1448-1454

9 Kalaivanam KN, Dharmalingam M, Marcus SR. Oxidative protein and lipid damage in type 2 diabetes mellitus. Paper presented at: International Conference on "Antioxidants and Free Radicals in Health-Nutrition \& Radio-Protectors" 4th Annual Conference of Society for Free Radical Research in India; 2005; Bangalore, India

10 Kalaivanam KN, Dharmalingam M, Marcus SR. Lipid peroxidation in type 2 diabetes mellitus. Int J Diabetes Dev Countries 2006;26:30-32

11 Dharmalingam M, Dev N, Marcus SR. High-sensitivity C-reactive protein levels in obese women. Paper presented at: 7th International Diabetes Federation Western Pacific Region Congress; 2008; Wellington, New Zealand

12 Veigas N, Dharmalingam M, Marcus SR. Oxidative stress in obesity and metabolic syndrome in Asian Indians. J Med Biochem 2011;30(2):115-120

13 Fernández_Real JM, López-Bermejo A, Ricart W. Crosstalk between iron metabolism and diabetes. Diabetes 2002;51(8):2348-2354

14 Ganesh S, Dharmalingam M, Marcus SR. Oxidative stress in type 2 diabetes with iron deficiency in Asian Indians. J Med Biochem 2012;31(2):115-120

15 Marcus SR, Dharmalingam M, Iron, oxidative stress and Diabetes. In: Preedy VR, ed. Diabetes: Oxidative Stress and Dietary Antioxidants. Oxford, United Kingdom: Elsevier; 2014:51-64

16 Griesmacher A, Kindhauser M, Andert SE, et al. Enhanced serum levels of thiobarbituric-acid-reactive substances in diabetes mellitus. Am J Med 1995;98(5):469-475

17 Evans JL, Goldfine ID, Maddux BA, Grodsky GM. Are oxidative stress-activated signaling pathways mediators of insulin resistance and $\beta$-cell dysfunction? Diabetes 2003;52(1):1-8

18 Ma X, Chen Z, Wang L, et al. The pathogenesis of diabetes mellitus by oxidative stress and inflammation: its inhibition by berberine. Front Pharmacol 2018;9:782

19 Crook M. Type 2 diabetes mellitus: a disease of the innate immune system? An update. Diabet Med 2004;21(3):203-207

20 Mahmoud F, Al-Ozairi E. Inflammatory cytokines and the risk of cardiovascular complications in type 2 diabetes. Dis Markers 2013;35(4):235-241

21 Donath MY. Targeting inflammation in the treatment of type 2 diabetes. Diabetes Obes Metab 2013;15(Suppl 3):193-196 